\title{
Synthesis and Biological Activity of Hydrazones of o- and p-Hydroxybenzoic Acids. Spatial Structure of 5-Bromo-2- hydroxybenzylidene-4- hydroxybenzohydrazide
}

\section{Authors: O. A. Nurkenova, Zh. B. Satpaevaa, Igor A. Schepetkin, A. I. Khlebnikovd, K. M. Turdybekov, T. M. Seilkhanov, and S. D. Fazylov}

This is a postprint of an article that originally appeared in Russian Journal of General Chemistry in October 2017. The final version can be found at https://dx.doi.org/10.1134/S1070363217100097.

Nurkenov, O. A. , Zh. B. Satpaeva, Igor A. Schepetkin, A. I. Khlebnikov, K. M. Turdybekov, T. M. Seilkhanov, and S. D. Fazylov. "Synthesis and Biological Activity of Hydrazones of o- and pHydroxybenzoic Acids. Spatial Structure of 5-Bromo-2-hydroxybenzylidene-4hydroxybenzohydrazide." Russian Journal of General Chemistry 87, no. 10 (October 2017): 2299-2306. DOI: 10.1134/S1070363217100097. 


\title{
Synthesis and Biological Activity of Hydrazones of $o$ - and $p$-Hydroxybenzoic Acids. Spatial Structure of 5-Bromo-2-hydroxybenzylidene-4-hydroxybenzohydrazide
}

\author{
O. A. Nurkenov ${ }^{a}$, Zh. B. Satpaeva ${ }^{a}$, I. A. Schepetkin ${ }^{b, c}$, A. I. Khlebnikov ${ }^{d, e}$, \\ K. M. Turdybekov ${ }^{f}$, T. M. Seilkhanov ${ }^{g}$, and S. D. Fazylov ${ }^{a}$ \\ ${ }^{a}$ Institute of Organic Synthesis and Coal Chemistry of Kazakhstan, \\ ul. Alikhanova 1, Karaganda, 100008 Kazakhstan \\ *e-mail: nurkenov_oral@mail.ru \\ ${ }^{b}$ Montana State University, Bozeman, USA \\ ${ }^{c}$ Russian-Speaking Academic Science Association (RASA) Center, \\ National Research Tomsk Polytechnic University, Tomsk, Russia \\ ${ }^{d}$ National Research Tomsk Polytechnic University, Tomsk, Russia \\ ${ }^{e}$ Polzunov Altai State Technical University, Barnaul, Russia \\ ${ }^{f}$ Buketov Karaganda State University, Karaganda, Kazakhstan \\ ${ }^{g}$ Ualikhanov Kokshetau State University, Kokshetau, Kazakhstan
}

\begin{abstract}
A series of hydrazones based on hydrazides of $o$ - and $p$-hydroxybenzoic acids have been prepared. $\mathrm{N}$-(5-Bromo-2-hydroxybenzylidene)-4-hydroxybenzohydrazide has been studied by X-ray diffraction analysis; its molecule forms hydrogen bond with a solvating ethanol molecule. Biological activity of the synthesized hydrazones towards cathepsin $\mathrm{E}$ and(or) elastase of human neutrophils has been determined.
\end{abstract}

Keywords: hydrazone, hydroxybenzoic acid hydrazide, cathepsin E, neutrophil elastase, molecular docking

DOI: $10.1134 / \mathrm{S} 1070363217100097$

The interest to hydrazones has recently emerged $[1,2]$ due to a broad range of their biological activity: antidepressant, antiinflammatory, antimalarial, antimicrobial, antiviral, and antitumor [3, 4]. For example, ftivazide, saluzide, and related drugs are used in the tuberculosis treatment $[5,6]$. Inhibitors of many enzymes like cyclooxygenase, monoaminoxidase, epoxide hydrolase, and various cathepsins have been prepared based on hydrazones [7-11]. Proteolytic enzymes cathepsin E (EC 3.4.23.34) and elastase of neutrophil (EC 3.4.21.37) play important part in pathogenesis of autoimmune diseases and tumors growth [12-14]. In view of the above, this study aimed to synthesize the derivatives of $\mathrm{N}$-arylidene hydrazones and investigate their antiinflammatory activity in the cellular system in vitro as well as the inhibiting activity with respect to proteolytic enzymes cathepsin $\mathrm{E}$ and elastase of neutrophil.

Hydrazones 3-16 were synthesized via the condensation of hydrazides of $o$ - and $p$-hydroxybenzoic acids
1 and $\mathbf{2}$ with a series of aromatic aldehydes (Scheme 1). The condensation reaction was performed by heating $\left(60-70^{\circ} \mathrm{C}\right)$ equimolar amounts of the aldehyde and the hydrazides in ethanol medium during 3-5 h. The target compounds were obtained with $70-90 \%$ yield.

The products 3-16 were readily crystallizable white substances, readily soluble in many organic solvents.

The IR spectra of $N$-arylidene hydrazones of $o$ - and p-hydroxybenzoic acids 3-16 did not contain bands of the $\mathrm{NH}_{2}$ group vibrations. The bands of the $\mathrm{NH}$ group stretching vibrations were observed at $3285-3355 \mathrm{~cm}^{-1}$. The hydrazone $\mathrm{C}=\mathrm{O}$ stretching vibrations were found at $1675-1690 \mathrm{~cm}^{-1}$. A group of characteristic bands at $1600-1440 \mathrm{~cm}^{-1}$ was assigned to the stretching vibrations of the aromatic ring.

The downfield part of the ${ }^{1} \mathrm{H}$ NMR spectrum of 2-hydroxy- $N$-(2-hydroxybenzylidene)benzohydrazide 6 contained the signals of methine protons of the 


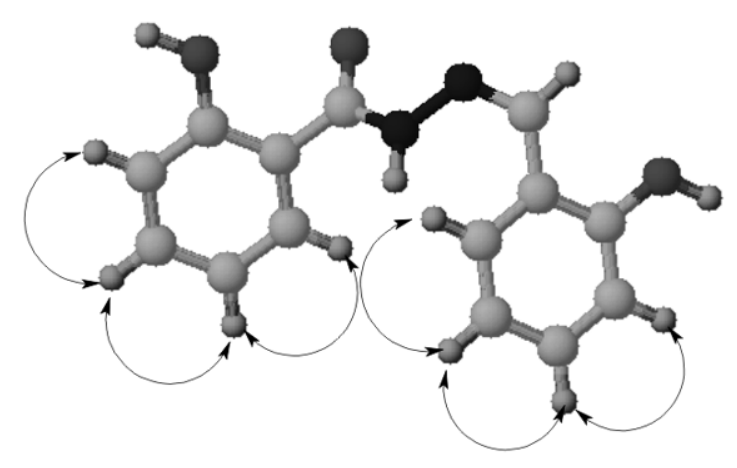

Fig. 1. $\operatorname{COSY}{ }^{1} \mathrm{H}-{ }^{1} \mathrm{H}$ correlations in compound 6 .

aromatic systems: $6.74-6.90\left(\mathrm{H}^{4}, \mathrm{H}^{6}, \mathrm{H}^{15}, \mathrm{H}^{17}\right), 7.26$ $\left(\mathrm{H}^{5}\right), 7.47\left(\mathrm{H}^{3}\right), 7.67\left(\mathrm{H}^{18}\right)$, and $7.79\left(\mathrm{H}^{16}\right) \mathrm{ppm}$. The most downfield signals $(10.14,11.40$, and $11.89 \mathrm{ppm})$ with integral intensity $1 \mathrm{H}$ were assigned to the protons of hydroxy and amino groups.<smiles>O=C(NN=Cc1ccccc1O)c1ccccc1O</smiles>

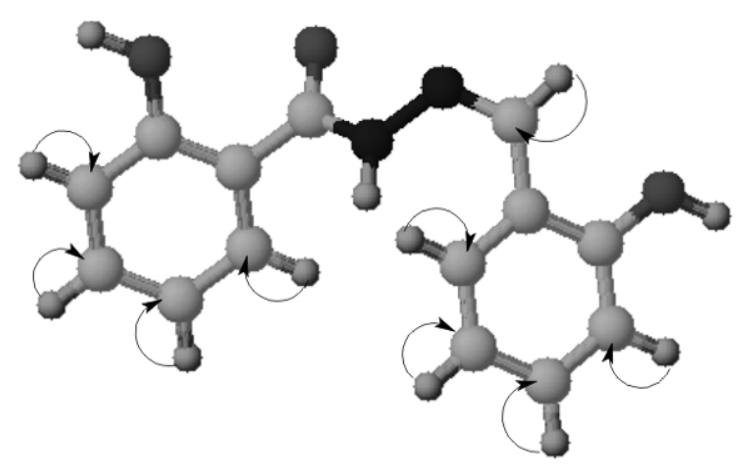

Fig. 2. $\mathrm{HMQC}{ }^{1} \mathrm{H}-{ }^{13} \mathrm{C}$ correlations in compound 6 .

The ${ }^{13} \mathrm{C}$ NMR spectrum of compound $\mathbf{6}$ contained the signals of the $\mathrm{CH}$ groups of two benzene rings at 115-132 ppm: $115.67\left(\mathrm{C}^{15}\right), 116.93\left(\mathrm{C}^{6}\right), 119.22\left(\mathrm{C}^{17}\right)$, $119.81\left(C^{4}\right), 128.63\left(C^{18}\right), 130.18\left(C^{3}\right), 130.27\left(C^{16}\right)$, and $131.66\left(\mathrm{C}^{5}\right) \mathrm{ppm}$. Quaternary atoms of the aromatic systems were assigned to the signals at $115.50\left(\mathrm{C}^{2}\right)$, $123.72\left(\mathrm{C}^{13}\right), 157.98\left(\mathrm{C}^{14}\right)$, and $161.46\left(\mathrm{C}^{1}\right) \mathrm{ppm}$. The signal at $148.18 \mathrm{ppm}$ corresponded to the $s p^{2}$-hybridized $\mathrm{C}^{12}$ atom. The downfield signal at $166.48 \mathrm{ppm}$ was assigned to the carbonyl atom $\mathrm{C}^{8}$.

The $\mathrm{H}-\mathrm{H}$ and $\mathrm{C}-\mathrm{H}$ spin-spin coupling in compound 6 via one or several bonds were observed by means of two-dimensional NMR spectroscopy COSY and HMQC (Figs. 1 and 2).

\section{Scheme 1.}

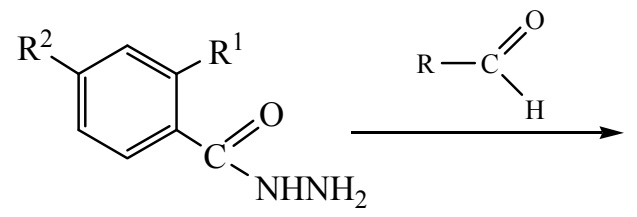

1,2<smiles>[R]C=NC(=O)c1ccc([R2])cc1[R]</smiles>

3-16

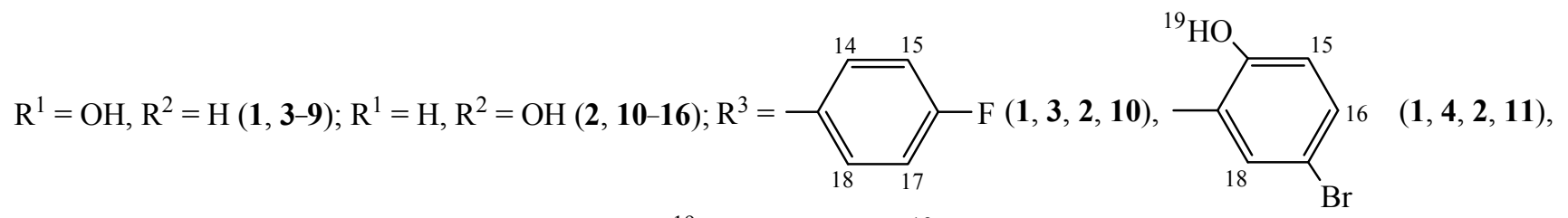<smiles>COc1ccc(C)cc1</smiles><smiles>Cc1ccncc1</smiles>

$(1,8,2,15)$,<smiles>CCOc1cc(C)ccc1O</smiles>

$(1,9,2,16)$ 
To elucidate the spatial structure of the derivatives of $\mathrm{N}$-arylidenehydrazones of $p$-hydroxybenzoic acid we performed the X-ray diffraction study of a solvate of $\mathrm{N}$-(5-bromo-2-hydroxybenzylidene)-4-hydroxybenzohydrazide 11 with ethanol (Fig. 3). The obtained data revealed that the bond lengths and bond angles in compound $\mathbf{1 1}$ were close to the standard ones [15]. The molecule of compound $\mathbf{1 1}$ was practically planar, but the phenyl cycles were slightly twisted with respect to each other (the dihedral angle was $5.9^{\circ}$ ). In the crystal each molecule of compound $\mathbf{1 1}$ formed the $\mathrm{N}^{1}-\mathrm{H} \cdots \mathrm{O}^{4}$ $(x, y, z)$ hydrogen bond $[\mathrm{N} \cdots \mathrm{O} 2.956(5), \mathrm{H} \cdots \mathrm{O} 2.22(4) \AA$, $\left.\mathrm{N}-\mathrm{H} \cdots \mathrm{O} 164(2)^{\circ}\right]$ with an ethanol molecule. Moreover, the $\mathrm{O}^{1}-\mathrm{H} \cdots \mathrm{O}^{2}$ intermolecular hydrogen bond was formed $[\mathrm{O} \cdots \mathrm{O} 2.243(4), \mathrm{H} \cdots \mathrm{O}$ 1.89(5) $\AA$, angle $\left.\mathrm{O}-\mathrm{H} \cdots \mathrm{O} 174(5)^{\circ}\right]$, leading to the organization of molecules of compound $\mathbf{1 1}$ in chains along the $a 0 c$ diagonal in parallel with the plane formed by that diagonal and the $b$ axis.

Compounds 3-16 were tested for antiinflammatory activity in vitro in a culture of human monocytic cells (MonoMac-6 cell line) reflected by the suppression of the lipopolysaccharide-induced production of cytokines interleukin-6 and tumor necrosis factor in the MonoMac-6 cells. The cells were treated with a solution of the tested compound in DMSO (final concentration of DMSO in the culture medium equaled $1 \%$ ) during $30 \mathrm{~min}$, and then bacterial lipopolysaccharide was added $(0.5 \mu \mathrm{g} / \mathrm{mL})$. The level of cytokines was evaluated by means of enzyme-linked immunosorbent assay after $24 \mathrm{~h}$ incubation of the MonoMac- 6 cells $\left(37^{\circ} \mathrm{C}\right.$, $5 \% \mathrm{CO}_{2}$ ). The obtained data revealed that none of the studied hydrazones suppressed the lipopolysaccharideinduced production of cytokines interleukin- 6 and tumor necrosis factor in the MonoMac-6 cells.

Various hydrazones exhibited the inhibiting activity towards certain proteases including cathepsins $[7,10$, 16]. Therefore, we evaluated the effect of the prepared compounds $3-16$ on the enzymatic activity of cathepsin $\mathrm{E}$ and neutrophil elastase. Since some of the tested compounds inhibited the enzymatic activity (see the table), to better reveal the structure-activity relationship we also studied the commercial analogs of the prepared hydrazones, 17-25 (Vitas-M Laboratory Ltd.). It was found that the compounds $4,17-19,21-$ 24 containing bromine $(4,17,21,22)$, chlorine (18), three hydroxyls $(\mathbf{1 9}, \mathbf{2 3})$, or four hydroxyls $(\mathbf{2 4})$ in the structure exhibited the activity towards cathepsin $\mathrm{E}$ $\left(\mathrm{IC}_{50}<50 \mu \mathrm{M}\right.$.). The highest activity was observed in the case of 5-bromo-2-hydroxy- $N^{\prime}$-[(1E)-(2-hydroxy-

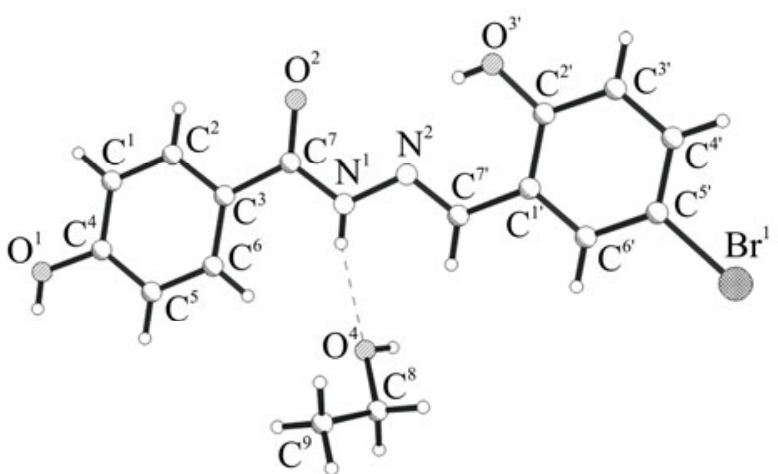

Fig. 3. General view of a molecule of ethanol solvate of $\mathrm{N}$-(5-bromo-2-hydroxybenzylidene)-4-hydroxybenzohydrazide 11.

phenyl)methylidene]benzohydrazide 22 ( $\left.\mathrm{IC}_{50} 14.4 \mu \mathrm{M}\right)$. Hence, the introduction of chlorine or bromine atoms in the structure of hydrazones can be considered a promising approach to prepare new inhibitors of cathepsin E.

The highest inhibiting activity with respect to the neutrophils elastase was observed for compound $\mathbf{2 5}$ $\left(\mathrm{IC}_{50} 21.1 \mu \mathrm{M}\right)$. Molecular docking of the active compounds in the structure of the neutrophils elastase [17] revealed that benzoyl groups of the active compounds 4 and 25 were located in the region of the central pocket of the active site of the elastase, similarly to the position of the co-crystallized peptide inhibitor of the elastase, but did not penetrate deeply in the pocket. The benzene rings adjacent to the imine fragment of the hydrazones were located in the wide part of the hydrophobic pocket, near the surface of the protein. Notably, the benzoyl fragments of molecules 4 and 25 formed three hydrogen bonds with Ser214 and Val216, the $\mathrm{OH}$ group acting as the donor and acceptor of the hydrogen bonds; this evidently facilitated the attaching of the molecules in the active center of the elastase favorable for the inhibition. This is exemplified in Scheme 2 by the location of the hydrogen bonds and hydrophobic interactions for hydrazone 25.

The positioning of the inactive hydrazones 3 and $\mathbf{6}$ in the active site of the neutrophils elastase were noticeably different from that of the active inhibitors. The benzene rings of molecules of compounds $\mathbf{3}$ and $\mathbf{6}$ penetrated deeply in the central hydrophobic pocket of the enzyme.

In summary, the condensation of hydrazides of $o$ and $p$-hydroxybenzoic acids with different aromatic aldehydes afforded a series of hydrazones, some of 
Inhibiting activity of the hydrazone towards cathepsin $\mathrm{E}$ and elastase of human neutrophils<smiles>[R]c1cc([R])c(C(=O)N/N=C/c2cc([R])c([R])c([R])c2[R])cc1[R]</smiles><smiles>O=C(N/N=C/c1ccncc1)c1cccc(O)c1</smiles>

3-7, 9-14, 16-25

$8(o-\mathrm{OH}), \mathbf{1 5}(p-\mathrm{OH})$

\begin{tabular}{|c|c|c|c|c|c|c|c|c|c|}
\hline \multirow{2}{*}{$\begin{array}{c}\text { Comp. } \\
\text { no. }\end{array}$} & \multirow{2}{*}{$\mathrm{R}^{1}$} & \multirow{2}{*}{$\mathrm{R}^{2}$} & \multirow{2}{*}{$\mathrm{R}^{3}$} & \multirow{2}{*}{$\mathrm{R}^{4}$} & \multirow{2}{*}{$\mathrm{R}^{5}$} & \multirow{2}{*}{$\mathrm{R}^{6}$} & \multirow{2}{*}{$\mathrm{R}^{7}$} & Cathepsin E & Elastase \\
\hline & & & & & & & & \multicolumn{2}{|c|}{$\mathrm{IC}_{50}, \mu \mathrm{M}^{\mathrm{a}}$} \\
\hline 3 & $\mathrm{H}$ & $\mathrm{H}$ & $\mathrm{OH}$ & $\mathrm{H}$ & $\mathrm{H}$ & $\mathrm{F}$ & $\mathrm{H}$ & - & - \\
\hline 4 & $\mathrm{H}$ & $\mathrm{H}$ & $\mathrm{OH}$ & $\mathrm{OH}$ & $\mathrm{H}$ & $\mathrm{H}$ & $\mathrm{Br}$ & 49.9 & 33.3 \\
\hline 5 & $\mathrm{H}$ & $\mathrm{H}$ & $\mathrm{OH}$ & $\mathrm{H}$ & $\mathrm{H}$ & $\mathrm{OCH}_{3}$ & $\mathrm{H}$ & - & - \\
\hline 6 & $\mathrm{H}$ & $\mathrm{H}$ & $\mathrm{OH}$ & $\mathrm{OH}$ & $\mathrm{H}$ & $\mathrm{H}$ & $\mathrm{H}$ & - & - \\
\hline 7 & $\mathrm{H}$ & $\mathrm{H}$ & $\mathrm{OH}$ & $\mathrm{H}$ & $\mathrm{H}$ & $\mathrm{OH}$ & $\mathrm{H}$ & 54.9 & 97.8 \\
\hline 8 & & - & - & & & & & & \\
\hline 9 & $\mathrm{H}$ & $\mathrm{H}$ & $\mathrm{OH}$ & $\mathrm{H}$ & $\mathrm{OCH}_{2} \mathrm{CH}_{3}$ & $\mathrm{OH}$ & $\mathrm{H}$ & - & 78.8 \\
\hline 10 & $\mathrm{H}$ & $\mathrm{OH}$ & $\mathrm{H}$ & $\mathrm{H}$ & $\mathrm{H}$ & $\mathrm{F}$ & $\mathrm{H}$ & - & - \\
\hline 11 & $\mathrm{H}$ & $\mathrm{OH}$ & $\mathrm{H}$ & $\mathrm{OH}$ & $\mathrm{H}$ & $\mathrm{H}$ & $\mathrm{OH}$ & 60.8 & - \\
\hline 12 & $\mathrm{H}$ & $\mathrm{OH}$ & $\mathrm{H}$ & $\mathrm{H}$ & $\mathrm{H}$ & $\mathrm{OCH}_{3}$ & $\mathrm{H}$ & - & - \\
\hline 13 & $\mathrm{H}$ & $\mathrm{OH}$ & $\mathrm{H}$ & $\mathrm{OH}$ & $\mathrm{H}$ & $\mathrm{H}$ & $\mathrm{H}$ & 89.7 & - \\
\hline 14 & $\mathrm{H}$ & $\mathrm{OH}$ & $\mathrm{H}$ & $\mathrm{H}$ & $\mathrm{H}$ & $\mathrm{OH}$ & $\mathrm{H}$ & 66.5 & - \\
\hline 15 & & - & - & & & & & & \\
\hline 16 & $\mathrm{H}$ & $\mathrm{OH}$ & $\mathrm{H}$ & $\mathrm{H}$ & $\mathrm{OCH}_{2} \mathrm{CH}_{3}$ & $\mathrm{OH}$ & $\mathrm{H}$ & 94.2 & 64.0 \\
\hline 17 & $\mathrm{H}$ & $\mathrm{H}$ & $\mathrm{OH}$ & $\mathrm{H}$ & $\mathrm{H}$ & $\mathrm{H}$ & $\mathrm{Br}$ & 34.2 & - \\
\hline 18 & $\mathrm{H}$ & $\mathrm{H}$ & $\mathrm{OH}$ & $\mathrm{OH}$ & $\mathrm{H}$ & $\mathrm{H}$ & $\mathrm{Cl}$ & 21.9 & 80.8 \\
\hline 19 & $\mathrm{H}$ & $\mathrm{H}$ & $\mathrm{OH}$ & $\mathrm{OH}$ & $\mathrm{OH}$ & $\mathrm{H}$ & $\mathrm{H}$ & 36.7 & - \\
\hline 20 & $\mathrm{H}$ & $\mathrm{H}$ & $\mathrm{OH}$ & $\mathrm{OH}$ & $\mathrm{H}$ & $\mathrm{OH}$ & $\mathrm{H}$ & 59.0 & - \\
\hline 21 & $\mathrm{H}$ & $\mathrm{H}$ & $\mathrm{OH}$ & $\mathrm{OH}$ & $\mathrm{H}$ & $\mathrm{OH}$ & $\mathrm{Br}$ & 20.1 & 60.4 \\
\hline 22 & $\mathrm{Br}$ & $\mathrm{H}$ & $\mathrm{OH}$ & $\mathrm{OH}$ & $\mathrm{H}$ & $\mathrm{H}$ & $\mathrm{H}$ & 14.4 & - \\
\hline 23 & $\mathrm{OH}$ & $\mathrm{H}$ & $\mathrm{H}$ & $\mathrm{OH}$ & $\mathrm{H}$ & $\mathrm{H}$ & $\mathrm{OH}$ & 32.3 & - \\
\hline 24 & $\mathrm{OH}$ & $\mathrm{H}$ & $\mathrm{OH}$ & $\mathrm{OH}$ & $\mathrm{H}$ & $\mathrm{OH}$ & $\mathrm{H}$ & 33.5 & 42.3 \\
\hline 25 & $\mathrm{H}$ & $\mathrm{H}$ & $\mathrm{OH}$ & $\mathrm{H}$ & $\mathrm{H}$ & $\mathrm{N}\left(\mathrm{CH}_{2} \mathrm{CH}_{2} \mathrm{CH}_{3}\right)_{2}$ & $\mathrm{H}$ & 79.2 & 21.1 \\
\hline
\end{tabular}

${ }^{\mathrm{a}}(-)$ the enzyme activity is not suppressed at the substrate concentration $<100 \mu \mathrm{M}$.

them were found promising for the synthesis of new inhibitors of cathepsin E.

\section{EXPERIMENTAL}

${ }^{1} \mathrm{H}$ and ${ }^{13} \mathrm{C}$ spectra were recorded using a JNN-ECA Jeol 400 spectrometer (399.78 and $100.53 \mathrm{MHz}$, respec- tively) in DMSO- $d_{6}$. Chemical shifts were measured relative to residual protons or carbon atoms of the solvent. The reaction course and the purity of the products were monitored by means of thin-layer chromatography on Silufol UV-254 plates (isopropanol-benzeneammonia, $10: 5: 2$, developing in iodine vapor). 


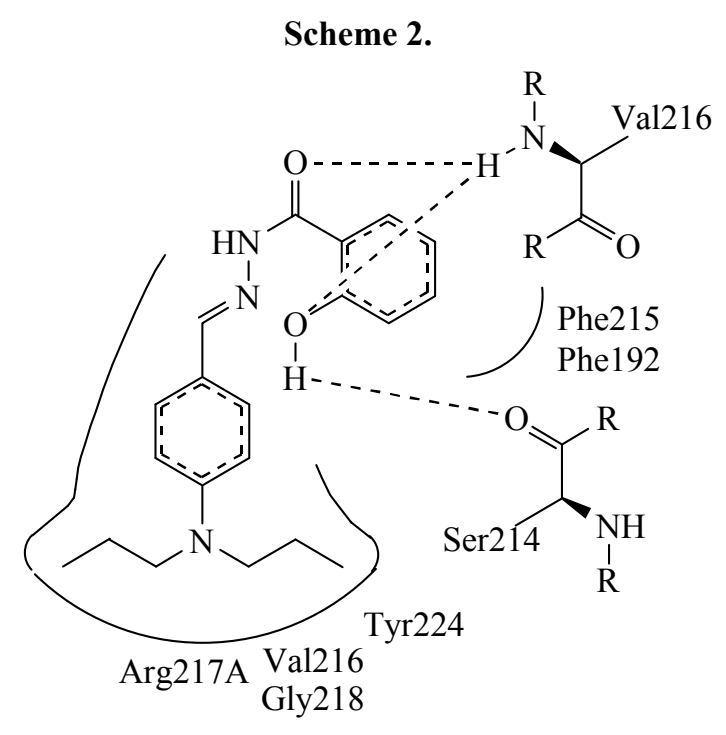

X-ray diffraction study of compound 11. The intensities of 6311 reflections (3293 independent ones, $\mathrm{R}_{\text {int }}$ 0.0516) were measured using an Xcalibur Ruby diffractometer (Oxford Diffraction) $\left(\mathrm{Cu} K_{\alpha}\right.$, graphite monochromator, $\varphi, \theta$-scanning, $\left.4.03 \leq \theta \leq 76.13^{\circ}\right)$ at $293 \mathrm{~K}$. The crystals were monoclinic, $\mathrm{C}_{14} \mathrm{H}_{11} \mathrm{~N}_{2} \mathrm{O}_{3} \mathrm{Br}$. $\mathrm{C}_{2} \mathrm{H}_{5} \mathrm{OH}, a$ 18.233(4), $b$ 17.857(4), c 13.191(3) $\AA, \beta$ $130.31(3)^{\circ}, V 3275(1) \AA^{3}, Z 8$, space group $C 2 / c, d_{\text {calc }}$ $1.546 \mathrm{~g} / \mathrm{cm}^{3}, \mu 3.618 \mathrm{~mm}^{-1}$. The dataset was processed (including the accounting for absorption) using SAINT and SADABS software.

Structure of compound $\mathbf{1 1}$ was solved via the direct method. Positions of the non-hydrogen atoms were refined in the anisotropic approximation by full-matrix least-squares method. The hydrogen atoms at $\mathrm{N}^{1}, \mathrm{O}^{1}$, $\mathrm{O}^{3^{\prime}}$, and $\mathrm{O}^{4}$ were found from differential synthesis, and their positions were refined in the isotropic approximation. Other hydrogen atoms were put in the geometry defined positions and refined in the isotropic approximation with fixed position and thermal parameters using the rider model. The structure was solved and refined using SHELXS-97 and SHELXL97 software packages [18, 19]. 1750 independent reflections with $I \geq 2 \sigma(I)$ were used in the refinement, 225 parameters were refined. The final divergence factors were as follows: $R_{1} 0.0501,{ }_{W} R_{2} 0.0938$ [over reflections with $I \geq 2 \sigma(I)], R_{1} 0.1085,{ }_{W} R_{2} 0.1190$ (over all reflections), GooF 0.969. Peaks of residual electronic density: $\Delta \rho 0.258$ and $-0.430 e / \AA^{3}$. Crystallographic data were deposited at the Cambridge Crystallographic Data Centre (CCDC 1546889).

$\mathrm{N}$-(4-Fluorobenzylidene)-2-hydroxybenzohydrazide (3). $p$-Fluorobenzaldehyde $(0.63 \mathrm{~g}, 0.0051 \mathrm{~mol})$ was added to a stirred solution of $0.76 \mathrm{~g}(0.005 \mathrm{~mol})$ of $o$-hydroxybenzoic acid hydrazide in $10 \mathrm{~mL}$ of 2-propanol. The mixture was stirred during $30 \mathrm{~min}$ at $60-70^{\circ} \mathrm{C}$. The reaction completeness was monitored by means of TLC. After cooling the mixture to ambient the fine crystalline precipitate was filtered off and recrystallized. Yield $1.08 \mathrm{~g}(83.8 \%), \mathrm{mp} 250-251^{\circ} \mathrm{C}(1,4-$ dioxane-hexane, $1: 2)$. ${ }^{1} \mathrm{H}$ NMR spectrum, $\delta$, ppm: $6.23 \mathrm{~d}\left(1 \mathrm{H}, \mathrm{CH}_{\mathrm{Ar}}^{1},{ }^{2} J_{\mathrm{HH}}=7.5 \mathrm{~Hz}\right), 6.62 \mathrm{t}\left(1 \mathrm{H}, \mathrm{CH}_{\mathrm{Ar}}{ }_{\mathrm{Ar}}\right.$, $\left.J_{\mathrm{HH}}=8.4 \mathrm{~Hz}\right), 6.45 \mathrm{t}\left(1 \mathrm{H}, \mathrm{CH}_{\mathrm{Ar}}^{3},{ }^{2} J_{\mathrm{HH}}=8.5 \mathrm{~Hz}\right), 6.77 \mathrm{t}$ $\left(1 \mathrm{H}, \mathrm{CH}_{\mathrm{Ar}}^{4},{ }^{2} J_{\mathrm{HH}}=7.3 \mathrm{~Hz}\right), 7.74 \mathrm{~d}\left(2 \mathrm{H}, \mathrm{CH}^{15,17} \mathrm{Ar},{ }^{2} J_{\mathrm{HH}}=\right.$ $8.6 \mathrm{~Hz}), 7.89 \mathrm{~d}\left(2 \mathrm{H}, \mathrm{CH}^{14,18}{ }_{\mathrm{Ar}},{ }^{2} J_{\mathrm{HH}}=8.5 \mathrm{~Hz}\right), 8.57 \mathrm{~s}$ $(1 \mathrm{H}, \mathrm{N}=\mathrm{CH}), 10.45 \mathrm{~s}(1 \mathrm{H}, \mathrm{OH}), 11.85 \mathrm{~s}(1 \mathrm{H}, \mathrm{N} \underline{\mathrm{H}}-\mathrm{N})$.

Hydrazides 4-16 were prepared similarly.

$\mathrm{N}$-(5-Bromo-2-hydroxybenzylidene)-2-hydroxybenzohydrazide (4). Yield $1.35 \mathrm{~g}(80 \%), \mathrm{mp} 295^{\circ} \mathrm{C}$. ${ }^{1} \mathrm{H}$ NMR spectrum, $\delta$, ppm: $6.90 \mathrm{~d}\left(1 \mathrm{H}, \mathrm{CH}^{15}{ }_{\mathrm{Ar}},{ }^{2} J_{\mathrm{HH}}=\right.$ $8.8 \mathrm{~Hz}), 6.95 \mathrm{t}\left(1 \mathrm{H}, \mathrm{CH}^{2}{ }_{\mathrm{Ar}}, J_{\mathrm{HH}}=7.4 \mathrm{~Hz}\right), 7.02 \mathrm{~d}(1 \mathrm{H}$, $\left.\mathrm{CH}_{\mathrm{Ar}}^{1}, J_{\mathrm{HH}}=8.0 \mathrm{~Hz}\right), 7.32 \mathrm{~d}\left(1 \mathrm{H}, \mathrm{CH}_{\mathrm{Ar}}^{4}, J_{\mathrm{HH}}=\right.$ $7.6 \mathrm{~Hz}), 7.42 \mathrm{t}\left(1 \mathrm{H}, \mathrm{CH}_{\mathrm{Ar}}^{3}, J_{\mathrm{HH}}=8.4 \mathrm{~Hz}\right), 7.42 \mathrm{~d}(1 \mathrm{H}$, $\left.\mathrm{CH}^{16}{ }_{\mathrm{Ar}}, J_{\mathrm{HH}}=8.7 \mathrm{~Hz}\right), 7.76 \mathrm{~s}\left(1 \mathrm{H}, \mathrm{CH}_{\mathrm{Ar}}\right), 8.57 \mathrm{~s}(1 \mathrm{H}$, $\mathrm{N}=\mathrm{CH}), 10.32 \mathrm{~s}\left(1 \mathrm{H}, \mathrm{OH}^{7}\right), 11.42 \mathrm{~s}(1 \mathrm{H}, \mathrm{OH}), 11.98 \mathrm{~s}$ $(1 \mathrm{H}, \mathrm{N} \underline{\mathrm{H}}-\mathrm{N})$.

2-Hydroxy- $\mathrm{N}$-(4-methoxybenzylidene)benzohydrazide (5). Yield $1.12 \mathrm{~g} \mathrm{(83 \% ),} \mathrm{mp} 215-218^{\circ} \mathrm{C}$ (2-propanol). ${ }^{1} \mathrm{H}$ NMR spectrum, $\delta$, ppm: $3.76 \mathrm{~s}\left(3 \mathrm{H}, \mathrm{OCH}_{3}\right)$, $6.26 \mathrm{~d}\left(1 \mathrm{H}, \mathrm{CH}_{\mathrm{Ar}}^{1}, J_{\mathrm{HH}}=7.5 \mathrm{~Hz}\right), 6.48 \mathrm{t}\left(1 \mathrm{H}, \mathrm{CH}_{\mathrm{Ar}}^{3}\right.$, $\left.J_{\mathrm{HH}}=8.5 \mathrm{~Hz}\right), 6.65 \mathrm{t}\left(1 \mathrm{H}, \mathrm{CH}^{2}{ }_{\mathrm{Ar}}, J_{\mathrm{HH}}=8.4 \mathrm{~Hz}\right), 6.80 \mathrm{~d}$ $\left(1 \mathrm{H}, \mathrm{CH}_{\mathrm{Ar}}^{4}, J_{\mathrm{HH}}=7.1 \mathrm{~Hz}\right), 6.84 \mathrm{~d}\left(2 \mathrm{H}, \mathrm{CH}^{15,17} \mathrm{Ar},{ }^{3} J_{\mathrm{HH}}=\right.$ $8.7 \mathrm{~Hz}), 7.60 \mathrm{~d}\left(2 \mathrm{H}, \mathrm{CH}^{14,18} \mathrm{Ar},{ }^{3} J_{\mathrm{HH}}=8.7 \mathrm{~Hz}\right), 8.35 \mathrm{~s}$ $(1 \mathrm{H}, \mathrm{N}=\mathrm{CH}), 10.01 \mathrm{~s}(1 \mathrm{H}, \mathrm{OH}), 11.49 \mathrm{~s}(1 \mathrm{H}, \mathrm{NH}) .{ }^{13} \mathrm{C}$ NMR spectrum, $\delta_{\mathrm{C}}$, ppm: $55.81\left(\mathrm{OCH}_{3}\right), 114.84$ $\left(\mathrm{CH}^{2,6}{ }_{\mathrm{Ar}}\right), 115.51\left(\mathrm{CH}^{15,17} \mathrm{Ar}\right), 119.81\left(\mathrm{C}_{\mathrm{Ar}}^{4}\right), 129.06$ $\left(\mathrm{CH}^{14,18}{ }_{\mathrm{Ar}}\right), 130.11\left(\mathrm{CH}_{\mathrm{Ar}}^{3,5}\right), 147.36(\mathrm{~N}=\mathrm{CH}), 161.21$ $\left(\mathrm{C}_{\mathrm{Ar}}^{1}, \mathrm{C}^{16}{ }_{\mathrm{Ar}}\right), 163.21(\mathrm{C}=\mathrm{O})$.

2-Hydroxy- $\mathrm{N}$-(2-hydroxybenzylidene)benzohydrazide (6). Yield $1.15 \mathrm{~g}$ (90\%), mp $273-275^{\circ} \mathrm{C}$ (EtOH). ${ }^{1} \mathrm{H}$ NMR spectrum, $\delta$, ppm: 6.84-6.90 m $(4 \mathrm{H}$, $\left.\mathrm{CH}^{4,6,15,17} \mathrm{Ar}\right), 7.26 \mathrm{t}\left(1 \mathrm{H}, \mathrm{CH}^{5} \mathrm{Ar}^{3} J_{\mathrm{HH}}=7.3 \mathrm{~Hz}\right), 7.47 \mathrm{~d}$ $\left(1 \mathrm{H}, \mathrm{CH}_{\mathrm{Ar}}^{3},{ }^{3} J_{\mathrm{HH}}=8.7 \mathrm{~Hz}\right), 7.67 \mathrm{~d}\left(1 \mathrm{H}, \mathrm{CH}^{18}{ }_{\mathrm{Ar}},{ }^{3} J_{\mathrm{HH}}=\right.$ $7.8 \mathrm{~Hz}), 7.79 \mathrm{t}\left(1 \mathrm{H}, \mathrm{CH}^{16}{ }_{\mathrm{Ar}},{ }^{3} \mathrm{~J}_{\mathrm{HH}}=8.2 \mathrm{~Hz}\right), 8.56 \mathrm{~s}(1 \mathrm{H}$, $\mathrm{N}=\mathrm{CH}), 10.14 \mathrm{~s}\left(1 \mathrm{H}, \mathrm{OH}^{19}\right), 11.40 \mathrm{~s}\left(1 \mathrm{H}, \mathrm{OH}^{7}\right), 11.89$ $\mathrm{s}(1 \mathrm{H}, \mathrm{NH}) .{ }^{13} \mathrm{C}$ NMR spectrum, $\delta_{\mathrm{C}}, \mathrm{ppm}: 115.12$ $\left(\mathrm{C}_{\mathrm{Ar}}^{2}\right), \quad 115.67 \quad\left(\mathrm{CH}^{15}{ }_{\mathrm{Ar}}\right), 116.93 \quad\left(\mathrm{CH}_{\mathrm{Ar}}^{6}\right), 119.22$ $\left(\begin{array}{lllll}\mathrm{C}^{17} \mathrm{Ar} & \end{array}\right), 119.81 \quad\left(\mathrm{CH}_{\mathrm{Ar}}^{4}\right), \quad 123.72 \quad\left(\mathrm{C}_{\mathrm{Ar}}^{13}\right), 128.12$ $\left(\mathrm{CH}^{18}{ }_{\mathrm{Ar}}\right), 130.27\left(\mathrm{CH}^{16}{ }_{\mathrm{Ar}}, \mathrm{CH}^{3}{ }_{\mathrm{Ar}}\right), 131.66\left(\mathrm{CH}_{\mathrm{Ar}}^{5}\right)$, $148.18(\mathrm{~N}=\mathrm{CH}), 157.98\left(\mathrm{C}^{14}{ }_{\mathrm{Ar}}\right),\left(\mathrm{C}^{1}{ }_{\mathrm{Ar}}\right), 166.48(\mathrm{C}=\mathrm{O})$.

2-Hydroxy- $\mathrm{N}$-(4-hydroxybenzylidene)hydrazide (7). Yield $1.06 \mathrm{~g}(83 \%), \mathrm{mp} 277-278^{\circ} \mathrm{C}(\mathrm{EtOH}) .{ }^{1} \mathrm{H}$ 
NMR spectrum, $\delta$, ppm: $6.83 \mathrm{~d}\left(2 \mathrm{H}, \mathrm{CH}^{15,17}{ }_{\mathrm{Ar}},{ }^{2} J_{\mathrm{HH}}=\right.$ $8.2 \mathrm{~Hz}), 6.92$ d.d $\left(2 \mathrm{H}, \mathrm{CH}^{4,6}{ }_{\mathrm{Ar}},{ }^{2} J_{\mathrm{HH}}=16.0,{ }^{3} J_{\mathrm{HH}}=\right.$ $7.6 \mathrm{~Hz}), 7.38 \mathrm{t}\left(1 \mathrm{H}, \mathrm{CH}_{\mathrm{Ar}}^{5},{ }^{2} J_{\mathrm{HH}}=7.8 \mathrm{~Hz}\right), 7.54 \mathrm{~d}(2 \mathrm{H}$, $\left.\mathrm{CH}^{14,18}{ }_{\mathrm{Ar}},{ }^{2} \mathrm{~J}_{\mathrm{HH}}=8.2 \mathrm{~Hz}\right), 7.86 \mathrm{~d}\left(1 \mathrm{H}, \mathrm{CH}^{3}{ }_{\mathrm{Ar}},{ }^{2} \mathrm{~J}_{\mathrm{HH}}=7.8 \mathrm{~Hz}\right)$, $8.33 \mathrm{~s}(\mathrm{~N}=\mathrm{CH}), 9.92$ br.s $\left(1 \mathrm{H}, \mathrm{OH}^{19}\right), 11.66 \mathrm{~s}(1 \mathrm{H}$, $\mathrm{NH}), 11.96$ br.s $\left(1 \mathrm{H}, \mathrm{OH}^{7}\right) .{ }^{13} \mathrm{C}$ NMR spectrum, $\delta_{\mathrm{C}}$, ppm: $\quad 116.20 \quad\left(\mathrm{C}_{\mathrm{Ar}}^{2}\right), \quad 116.29 \quad\left(\mathrm{CH}^{15,17}{ }_{\mathrm{Ar}}\right), \quad 117.85$ $\left(\mathrm{CH}_{\mathrm{Ar}}^{6}\right), \quad 119.38\left(\mathrm{CH}_{\mathrm{Ar}}^{4}\right), \quad 125.60\left(\mathrm{C}^{13}{ }_{\mathrm{Ar}}\right), 128.81$ $\left(\mathrm{CH}_{\mathrm{Ar}}^{3}\right), 129.62\left(\mathrm{CH}^{14,18}{ }_{\mathrm{Ar}}\right), 134.25\left(\mathrm{CH}_{\mathrm{Ar}}^{5}\right), 149.78$ $(\mathrm{N}=\mathrm{CH}), 159.85\left(\mathrm{C}^{16}{ }_{\mathrm{Ar}}\right), 160.21\left(\mathrm{C}^{1}{ }_{\mathrm{Ar}}\right), 165.25(\mathrm{C}=\mathrm{O})$.

2-Hydroxy- $N$-(pyridin-4-ylmethylidene)benzohydrazide (8). Yield $1.09 \mathrm{~g}$ (91\%), mp $238-239^{\circ} \mathrm{C}$ $(\mathrm{EtOH}) .{ }^{1} \mathrm{H}$ NMR spectrum, $\delta$, ppm: $6.95 \mathrm{~m}\left(2 \mathrm{H}, \mathrm{CH}^{4,6} \mathrm{Ar}\right)$, $7.41 \mathrm{t}\left(1 \mathrm{H}, \mathrm{CH}_{\mathrm{Ar}}^{5}, J_{\mathrm{HH}}=4.9 \mathrm{~Hz}\right), 7.63 \mathrm{~d}\left(2 \mathrm{H}, \mathrm{CH}^{14,18} \mathrm{Ar}\right.$, $\left.J_{\mathrm{HH}}=3.9 \mathrm{~Hz}\right), 7.84 \mathrm{~d}\left(1 \mathrm{H}, \mathrm{CH}_{\mathrm{Ar}}^{3}\right), 8.42 \mathrm{~s}(1 \mathrm{H}, \mathrm{N}=\mathrm{CH})$, $8.62 \mathrm{~d}\left(2 \mathrm{H}, \mathrm{CH}^{15,17} \mathrm{Ar}\right), 11.96$ br.s $(2 \mathrm{H}, \mathrm{NH}, \mathrm{OH}) .{ }^{13} \mathrm{C}$ NMR spectrum, $\delta_{\mathrm{C}}$, ppm: $116.83\left(\mathrm{C}_{\mathrm{Ar}}^{2}\right), 117.77$ $\left(\mathrm{CH}_{\mathrm{Ar}}^{6}\right), 119.61\left(\mathrm{C}_{\mathrm{Ar}}^{4}\right), 121.59\left(\mathrm{CH}^{14,18}{ }_{\mathrm{Ar}}\right), 129.46$ $\left(\mathrm{CH}_{\mathrm{Ar}}^{3}\right), 134.47\left(\mathrm{CH}_{\mathrm{Ar}}^{5}\right), 141.89\left(\mathrm{C}_{\mathrm{Ar}}^{13}\right), 146.63(\mathrm{~N}=\mathrm{CH})$, $150.81\left(\mathrm{CH}^{15,17}{ }_{\mathrm{Ar}}\right), 159.17\left(\mathrm{C}_{\mathrm{Ar}}^{1}\right), 165.34(\mathrm{C}=\mathrm{O})$.

$\mathrm{N}$-(3-Ethoxy-4-hydroxybenzylidene)-2-hydroxybenzohydrazide (9). Yield $0.9 \mathrm{~g}(60.6 \%)$, mp 196$198^{\circ} \mathrm{C}\left(\mathrm{C}_{6} \mathrm{H}_{6}\right) .{ }^{1} \mathrm{H}$ NMR spectrum, $\delta$, ppm: $1.33 \mathrm{t}(3 \mathrm{H}$, $\left.\mathrm{CH}_{3},{ }^{3} J_{\mathrm{HH}}=6.9 \mathrm{~Hz}\right), 4.01-4.06 \mathrm{~m}\left(2 \mathrm{H}, \mathrm{OCH}_{2}\right), 6.84 \mathrm{~d}$ $\left(1 \mathrm{H}, \mathrm{CH}_{\mathrm{Ar}}^{3},{ }^{3} J_{\mathrm{HH}}=8.2 \mathrm{~Hz}\right), 6.94$ d.d $\left(2 \mathrm{H}, \mathrm{CH}^{18,20} \mathrm{Ar}\right.$, $\left.{ }^{3} J_{\mathrm{HH}}=7.6,13.5 \mathrm{~Hz}\right), 7.07 \mathrm{~d}\left(1 \mathrm{H}, \mathrm{CH}^{4}{ }_{\mathrm{Ar}},{ }^{3} J_{\mathrm{HH}}=\right.$ $8.2 \mathrm{~Hz}), 7.28 \mathrm{~s}\left(1 \mathrm{H}, \mathrm{CH}_{\mathrm{Ar}}^{6}\right), 7.34-7.41 \mathrm{~m}\left(1 \mathrm{H}, \mathrm{CH}^{19}{ }_{\mathrm{Ar}}\right)$, 7.88 d.d $\left(1 \mathrm{H}, \mathrm{CH}^{21}{ }_{\mathrm{Ar}},{ }^{3} J_{\mathrm{HH}}=7.8,13.7 \mathrm{~Hz}\right), 8.30 \mathrm{~s}(1 \mathrm{H}$, $\mathrm{N}=\mathrm{CH})$.

$\mathrm{N}$-(4-Fluorobenzylidene)-4-hydroxybenzohydrazide (10). Yield $0.87 \mathrm{~g}(67.5 \%), \mathrm{mp} 183-185^{\circ} \mathrm{C}$ (propanol-2). ${ }^{1} \mathrm{H}$ NMR spectrum, $\delta$, ppm: $6.81 \mathrm{~d}(2 \mathrm{H}$, $\left.\mathrm{CH}^{2,6}{ }_{\mathrm{Ar}},{ }^{2} J=8.7 \mathrm{~Hz}\right), 7.78 \mathrm{t}\left(2 \mathrm{H}, \mathrm{CH}^{3,5}{ }_{\mathrm{Ar}}, J=8.7 \mathrm{~Hz}\right)$, $7.74 \mathrm{~d}\left(2 \mathrm{H}, \mathrm{CH}^{15,17} \mathrm{Ar},{ }^{2} J=8.6 \mathrm{~Hz}\right), 7.89 \mathrm{~d}(2 \mathrm{H}$, $\left.\mathrm{CH}^{14,18}{ }_{\mathrm{Ar}},{ }^{2} J=8.5 \mathrm{~Hz}\right), 8.58 \mathrm{~s}(1 \mathrm{H}, \mathrm{N}=\mathrm{CH}), 10.50 \mathrm{~s}$ $(1 \mathrm{H}, \mathrm{OH}), 11.83 \mathrm{~s}(1 \mathrm{H}, \mathrm{N} \underline{\mathrm{HN}})$.

$\mathrm{N}$-(5-Bromo-2-hydroxybenzylidene)-4-hydroxybenzohydrazide (11). Yield $0.85 \mathrm{~g}$ (51.1\%), mp 293$295^{\circ} \mathrm{C}(\mathrm{EtOH}) .{ }^{1} \mathrm{H}$ NMR spectrum, $\delta$, ppm: $6.88 \mathrm{~d}(2 \mathrm{H}$, $\left.\mathrm{CH}^{2,6}{ }_{\mathrm{Ar}},{ }^{2} J_{\mathrm{HH}}=8.7 \mathrm{~Hz}\right), 6.90 \mathrm{~d}\left(1 \mathrm{H}, \mathrm{CH}^{15}{ }_{\mathrm{Ar}}, J_{\mathrm{HH}}=\right.$ $8.8 \mathrm{~Hz}), 7.42 \mathrm{~d}\left(1 \mathrm{H}, \mathrm{CH}^{16}{ }_{\mathrm{Ar}} J_{\mathrm{HH}}=8.7 \mathrm{~Hz}\right), 7.76 \mathrm{~s}(1 \mathrm{H}$, $\left.\mathrm{CH}^{18}{ }_{\mathrm{Ar}}\right), 7.83 \mathrm{~d}\left(2 \mathrm{H}, \mathrm{CH}^{3,5}{ }_{\mathrm{Ar}}{ }^{2} J_{\mathrm{HH}}=8.5 \mathrm{~Hz}\right), 8.57 \mathrm{~s}$ $(1 \mathrm{H}, \mathrm{N}=\mathrm{CH}), 10.15 \mathrm{~s}\left(1 \mathrm{H}, \mathrm{OH}^{7}\right), 11.42 \mathrm{~s}(1 \mathrm{H}, \mathrm{OH})$, $11.98 \mathrm{~s}(1 \mathrm{H}, \mathrm{N} \underline{\mathrm{H}}-\mathrm{N})$.

4-Hydroxy- $\mathrm{N}$-(4-methoxybenzylidene)benzohydrazide (12). Yield $1.21 \mathrm{~g}(90 \%), \mathrm{mp} 220^{\circ} \mathrm{C}(\mathrm{EtOH})$. ${ }^{1} \mathrm{H}$ NMR spectrum, $\delta$, ppm: $3.76 \mathrm{~s}\left(3 \mathrm{H}, \mathrm{OCH}_{3}\right), 6.83 \mathrm{~d}$ $\left(2 \mathrm{H}, \mathrm{CH}^{15,17}{ }_{\mathrm{Ar}},{ }^{3} \mathrm{~J}_{\mathrm{HH}}=8.7 \mathrm{~Hz}\right), 6.97 \mathrm{~d}\left(2 \mathrm{H}, \mathrm{CH}^{2,6}{ }_{\mathrm{Ar}}\right.$, $\left.{ }^{3} J_{\mathrm{HH}}=8.7 \mathrm{~Hz}\right), 7.61 \mathrm{~d}\left(2 \mathrm{H}, \mathrm{CH}^{14,18}{ }_{\mathrm{Ar}},{ }^{3} J_{\mathrm{HH}}=8.7 \mathrm{~Hz}\right)$, $7.77 \mathrm{~d}\left(2 \mathrm{H}, \mathrm{CH}^{3,5}{ }_{\mathrm{Ar}},{ }^{3} J_{\mathrm{HH}}=7.8 \mathrm{~Hz}\right), 8.34 \mathrm{~s}(1 \mathrm{H}$, $\mathrm{N}=\mathrm{CH}), 10.03 \mathrm{~s}(1 \mathrm{H}, \mathrm{OH}), 11.46 \mathrm{~s}(1 \mathrm{H}, \mathrm{NH}) \cdot{ }^{13} \mathrm{C}$ NMR spectrum, $\delta_{\mathrm{C}}$, ppm: $55.80\left(\mathrm{OCH}_{3}\right), 114.85$ $\left(\mathrm{CH}^{2,6}{ }_{\mathrm{Ar}}\right), 115.51\left(\mathrm{CH}^{15,17} \mathrm{Ar}\right), 124.61\left(\mathrm{C}_{\mathrm{Ar}}^{4}\right), 127.64$ $\left(\mathrm{C}^{13} \mathrm{Ar}\right), 129.05\left(\mathrm{CH}^{14,18}{ }_{\mathrm{Ar}}\right), 130.11\left(\mathrm{CH}^{3,5} \mathrm{Ar}\right), 147.34$ $(\mathrm{N}=\mathrm{CH}), 161.21\left(\mathrm{C}^{1}{ }_{\mathrm{Ar}}, \mathrm{C}^{16}{ }_{\mathrm{Ar}}\right), 163.19(\mathrm{C}=\mathrm{O})$.

4-Hydroxy- $\mathrm{N}$-(2-hydroxybenzylidene)benzohydrazide (13). Yield $0.85 \mathrm{~g}(67 \%), \mathrm{mp} 260^{\circ} \mathrm{C}(\mathrm{EtOH})$. ${ }^{1} \mathrm{H}$ NMR spectrum, $\delta$, ppm: $6.85 \mathrm{~d}\left(2 \mathrm{H}, \mathrm{CH}^{2,6}{ }_{\mathrm{Ar}}, J_{\mathrm{HH}}=\right.$ $8.7 \mathrm{~Hz}), 6.90 \mathrm{~d}\left(2 \mathrm{H}, \mathrm{CH}^{15,17} \mathrm{Ar}, J_{\mathrm{HH}}=8.8 \mathrm{~Hz}\right), 7.74 \mathrm{~d}$ $\left(2 \mathrm{H}, \mathrm{CH}^{3,5}{ }_{\mathrm{Ar}}, J_{\mathrm{HH}}=7.8 \mathrm{~Hz}\right), 7.54 \mathrm{~d}\left(2 \mathrm{H}, \mathrm{CH}^{14,18}{ }_{\mathrm{Ar}}\right.$, $\left.{ }^{2} J_{\mathrm{HH}}=8.2 \mathrm{~Hz}\right), 8.28 \mathrm{~s}(1 \mathrm{H}, \mathrm{N}=\mathrm{CH}), 9.98$ br.s $(2 \mathrm{H}$, $\left.\mathrm{OH}^{7,19}\right), 11.40 \mathrm{~s}(1 \mathrm{H}, \mathrm{NH})$.

4-Hydroxy- $\mathrm{N}$-(4-hydroxybenzylidene)benzohydrazide (14). Yield $0.94 \mathrm{~g}(73.8 \%), \mathrm{mp} 265^{\circ} \mathrm{C}(\mathrm{EtOH})$. ${ }^{1} \mathrm{H}$ NMR spectrum, $\delta$, ppm: $6.80 \mathrm{~m}\left(4 \mathrm{H}, \mathrm{CH}^{2,6,15,17} \mathrm{Ar}\right)$, $7.50 \mathrm{~d}\left(2 \mathrm{H}, \mathrm{CH}^{14,18} \mathrm{Ar}, J_{\mathrm{HH}}=6.9 \mathrm{~Hz}\right), 7.74 \mathrm{~d}(2 \mathrm{H}$, $\left.\mathrm{CH}^{5,3}{ }_{\mathrm{Ar}}, J_{\mathrm{HH}}=8.2 \mathrm{~Hz}\right), 8.28 \mathrm{~s}(1 \mathrm{H}, \mathrm{N}=\mathrm{CH}), 9.95$ br.s $\left(2 \mathrm{H}, \mathrm{OH}^{7,19}\right), 11.40 \mathrm{~s}(1 \mathrm{H}, \mathrm{NH}) \cdot{ }^{13} \mathrm{C}$ NMR spectrum, $\delta_{\mathrm{C}}$, ppm: $115.49\left(\mathrm{CH}^{2,6}{ }_{\mathrm{Ar}}\right), 116.21\left(\mathrm{CH}^{15,17}{ }_{\mathrm{Ar}}\right), 124.63$ $\left(\mathrm{C}_{\mathrm{Ar}}^{4}\right), \quad 126.03 \quad\left(\mathrm{C}_{\mathrm{Ar}}^{13}\right), \quad 129.21 \quad\left(\mathrm{CH}^{14,18}{ }_{\mathrm{Ar}}\right), 130.07$ $\left(\mathrm{CH}^{5,3} \mathrm{Ar}\right), 147.74(\mathrm{~N}=\mathrm{CH}), 159.75\left(\mathrm{C}^{16}{ }_{\mathrm{Ar}}\right), 161.04\left(\mathrm{C}_{\mathrm{Ar}}^{1}\right)$, $163.07(\mathrm{C}=\mathrm{O})$.

4-Hydroxy- $\mathrm{N}$-(pyridin-4-ylmethylidene)benzohydrazide (15). Yield $1.07 \mathrm{~g}(89 \%), \mathrm{mp} 261-263^{\circ} \mathrm{C}(\mathrm{EtOH})$. ${ }^{1} \mathrm{H}$ NMR spectrum, $\delta$, ppm: $6.84 \mathrm{~d}\left(2 \mathrm{H}, \mathrm{CH}^{2,6}{ }_{\mathrm{Ar}}, J_{\mathrm{HH}}=\right.$ $8.9 \mathrm{~Hz}), 7.60 \mathrm{~d}\left(2 \mathrm{H}, \mathrm{CH}^{14,18} \mathrm{Ar}, J_{\mathrm{HH}}=6.0 \mathrm{~Hz}\right), 7.79 \mathrm{~d}$ $\left(2 \mathrm{H}, \mathrm{CH}^{3,5}{ }_{\mathrm{Ar}}, J_{\mathrm{HH}}=6.9 \mathrm{~Hz}\right), 8.37 \mathrm{~s}(\mathrm{~N}=\mathrm{CH}), 8.60 \mathrm{~d}$ $\left(2 \mathrm{H}, \mathrm{CH}^{15,17} \mathrm{Ar}, J_{\mathrm{HH}}=6.0 \mathrm{~Hz}\right), 10.12$ br.s $(1 \mathrm{H}, \mathrm{NH})$, $11.83 \mathrm{~s}(1 \mathrm{H}, \mathrm{OH}) .{ }^{13} \mathrm{C}$ NMR spectrum, $\delta_{\mathrm{C}}, \mathrm{ppm}$ : $115.62\left(\mathrm{CH}^{2,6}{ }_{\mathrm{Ar}}\right), 121.41\left(\mathrm{CH}^{14,18}{ }_{\mathrm{Ar}}\right), 124.07\left(\mathrm{C}_{\mathrm{Ar}}^{4}\right)$, $130.48\left(\mathrm{CH}^{3,5} \mathrm{Ar}\right), 142.26\left(\mathrm{C}^{13}{ }_{\mathrm{Ar}}\right), 144.83(\mathrm{~N}=\mathrm{CH})$, $150.75\left(\mathrm{CH}^{15,17}{ }_{\mathrm{Ar}}\right), 161.45\left(\mathrm{C}^{1}{ }_{\mathrm{Ar}}\right)$.

$\mathrm{N}$-(3-Ethoxy-4-hydroxybenzylidene)-4-hydroxybenzohydrazide (16). Yield $1.37 \mathrm{~g}$ (91.9\%), mp 240$242^{\circ} \mathrm{C}(\mathrm{EtOH}) .{ }^{1} \mathrm{H}$ NMR spectrum, $\delta$, ppm: $1.02 \mathrm{t}(3 \mathrm{H}$, $\left.\mathrm{CHCH}_{3},{ }^{3} J_{\mathrm{HH}}=6.9 \mathrm{~Hz}\right), 1.31 \mathrm{t}\left(3 \mathrm{H}, \mathrm{OCH}_{2} \mathrm{CH}_{3},{ }^{3} J_{\mathrm{HH}}=\right.$ $6.9 \mathrm{~Hz}), 3.33-3.43 \mathrm{~m}\left(1 \mathrm{H}, \mathrm{CHCH}_{3}\right), 3.93-4.04 \mathrm{~m}(2 \mathrm{H}$, $\left.\mathrm{OCH}_{2} \mathrm{CH}_{3}\right), 6.80 \mathrm{~d}\left(2 \mathrm{H}, \mathrm{CH}^{1 \overline{8,2} 0}{ }_{\mathrm{Ar}},{ }^{3} J_{\mathrm{HH}}=8.2 \mathrm{~Hz}\right), 6.82$ $\mathrm{d}\left(1 \mathrm{H}, \mathrm{CH}_{\mathrm{Ar}}^{3},{ }^{3} J_{\mathrm{HH}}=3.7 \mathrm{~Hz}\right), 7.03 \mathrm{~d}\left(1 \mathrm{H}, \mathrm{CH}^{4}{ }_{\mathrm{Ar}},{ }^{3} \mathrm{~J}_{\mathrm{HH}}=\right.$ $7.8 \mathrm{~Hz}), 7.24 \mathrm{~s}\left(1 \mathrm{H}, \mathrm{CH}_{\mathrm{Ar}}^{6}\right), 7.77 \mathrm{~d}\left(2 \mathrm{H}, \mathrm{CH}^{17,21}{ }_{\mathrm{Ar}},{ }^{3} J_{\mathrm{HH}}=\right.$ $8.7 \mathrm{~Hz}), 8.27 \mathrm{~s}(1 \mathrm{H}, \mathrm{N}=\mathrm{CH}) .{ }^{13} \mathrm{C}$ NMR spectrum, $\delta_{\mathrm{C}}$, ppm: $15.24\left(\mathrm{OCH}_{2} \underline{\mathrm{CH}}_{3}\right), \quad 19.06\left(\mathrm{CHCH}_{3}\right), \quad 56.58$ $\left(\underline{\mathrm{CHCH}}{ }_{3}\right), 64.43\left(\mathrm{O}^{\mathrm{C}} \mathrm{H}_{2} \mathrm{CH}_{3}\right), 110.87\left(\mathrm{CH}^{6}{ }_{\mathrm{Ar}}\right), 115.49$ $\left(\mathrm{CH}^{18,20}{ }_{\mathrm{Ar}}\right), 116.07\left(\mathrm{CH}^{4}{ }_{\mathrm{Ar}}\right), 122.42\left(\mathrm{CH}^{3}{ }_{\mathrm{Ar}}\right), 124.64$ $\left(\mathrm{C}_{\mathrm{Ar}}^{5}\right), \quad 126.47\left(\mathrm{C}^{16}{ }_{\mathrm{Ar}}\right), \quad 130.07 \quad\left(\mathrm{CH}^{17,21}{ }_{\mathrm{Ar}}\right), \quad 147.70$ $(\mathrm{N}=\mathrm{CH}), 149.60\left(\mathrm{C}_{\mathrm{Ar}}^{2}\right), 161.06\left(\mathrm{C}_{\mathrm{Ar}}^{1}\right), 163.15\left(\mathrm{C}^{19}{ }_{\mathrm{Ar}}\right)$. 
Activity with respect to elastase was estimated as the ability of the compound to inhibit hydrolysis of synthetic fluoro-genic substrate N-methylsuccinyl-AlaAla-Pro-Val-7-amino-4-methylcoumarin (Calbiochem) by elastase of human neutrophils (Calbiochem). The formation of the fluorescent product was detected under excitation at $355 \mathrm{~nm}$ from the intensity of emission at $460 \mathrm{~nm}$ using a Fluoroskan Ascent FL instrument (Thermo Scientific) [20]. Activity with respect to cathepsin E was estimated as the ability of the compound to inhibit hydrolysis of synthetic fluorogenic substrate of cathepsin E (1756.0 Da, Enzo Life Sciences, Inc.). The formation of the fluorescent product was detected under excitation at $325 \mathrm{~nm}$ from the intensity of emission at $410 \mathrm{~nm}$ using the same instrument.

Prior to determination of activity of cathepsin E, recombinant human cathepsin E (R\&D Systems Inc.) was incubated at room temperature during $30 \mathrm{~min}$ in $0.1 \mathrm{M}$ acetate buffer containing $0.5 \mathrm{M}$ of sodium chloride ( $\mathrm{pH}$ 3.5) until complete activation of the enzyme.

To measure the inhibiting activity of the compounds, $50 \mu \mathrm{L}$ of the enzyme (elastase or cathepsin E) $(0.2 \mu \mathrm{g} / \mathrm{mL}), 1 \mu \mathrm{L}$ of a solution of the tested compound in DMSO (the compound concentration was varied), and $50 \mu \mathrm{L}$ of the fluorogenic substrate solution $(40 \mu \mathrm{M})$ were put in a well of a 96 -well plate. The final concentration of DMSO in the reaction mixture equaled $1 \%$. Kinetics of fluorescence was monitored during $5 \mathrm{~min}$ after the mixing. The effective concentration of the compound suppressing the enzymatic activity by $50 \%\left(\mathrm{IC}_{50}\right)$ was determined by means of regression analysis.

Molecular docking. Structure of elastase from the 1HNE neutrophils containing a co-crystallized peptide inhibitor MeO-Suc-Ala-Ala-Pro-Ala chloromethyl ketone was obtained from the Protein Data Bank (PDB). The water molecules were removed from the 1HNE structure. The amino acid units within $6.5 \AA$ from the co-crystallized ligand were chosen as those constraining the active site of the elastase: Phe41, Cys42, Ala55, His57, Cys58, Tyr94, Pro98, Leu99B, Asp102, Val190, Cys191, Phe192, Gly193, Asp194, Ser195, Gly196, Ala213, Ser214, Phe215, Val216, Arg217A, Gly218, Gly219, and Tyr224. Docking of compounds $3,4,6$, and 25 was performed with account for their conformational lability and with rigid receptor using the default options of LeadIT software (BioSolveIT GmbH, Germany).

\section{ACKNOWLEDGMENTS}

This study was financially supported by the Ministry of Education and Science of Russian Federation (project no. 4.6660.2017/8.9).

\section{REFERENCES}

1. Mashkovskii, M.D., Lekarstvennyye sredstva (Drugs), Moscow: Novaya Volna, 2012.

2. Kulakov, I.V., Nurkenov, O.A., Satpaeva, Zh.B., and Turdybekov, K.M., Russ. J. Gen. Chem., 2014, vol. 84, no. 8, p. 1543. doi 10.1134/S1070363214080180

3. Rollas S., Küçükgüzel S.G., Molecules, 2007, vol. 12, no. 8, p. 1910. doi 10.3390/12081910

4. Lisina S.V., Brel A.K., Mazanova L.S., Spasov A.A., Pharm. Chem. J., 2008, vol. 42, no. 10, p. 574. doi 10.1007/S11094-009-0184-4.

5. Shchukina, M.N., Pershin, G.N., Sazonova, E.D., and Makeeva, O.O., Aromaticheskie izonikotinoilgidrazony novyi klass khimioterapevticheskikh protivotuberkuleznykh veshchestv. Khimiya i meditsina. Ftivazid (Aromatic isonicotinoyl hydrazones - a New Class of Chemotherapeutic anti-TB Drugs. Chemistry and Medicine. Phytivazide), Moscow: Medgiz, 1954, p. 12.

6. Maiocchi, S.L., Morris, J.C., Rees, M.D., and Thomas, S.R., Biochem. Pharm., 2017, vol. 135, p. 90. doi 10.1016/ j.bcp.2017.03.016

7. Evranos-Aksöz, B., Baysal, İ., Yabanoğlu-Çiftçi, S., Djikic, T., Yelekçi, K., Uçar, G., and Ertan, R., Arch. Pharm., 2015, vol. 348, no. 10, p. 743. doi 10.1002/ ardp. 201500212

8. Raghav, N. and Singh, M., Bioorg. Med. Chem., 2014, vol. 22, p. 4233. doi 10.1016/j.bmc.2014.05.037

9. Shen, H.C., Expert Opin. Ther. Pat., 2010, vol. 20, p. 941. doi 10.1517/13543776.2010.484804

10. Cywin, C.L., Firestone, R.A., McNeil, D.W., Grygon, C.A., Crane, K.M., White, D.M., Kinkade, P.R., Hopkins, J.L., Davidson, W., Labadia, M.E., Wildeson, J., Morelock, M.M., Peterson, J.D., Raymond, E.L., Brown, M.L., and Spero, D.M., Bioorg. Med. Chem., 2003, vol. 11, p. 733. doi 10.1016/S0968-0896(02)00468-6

11. Mohammed, K.O. and Nissan, Y.M., Chem. Biol. Drug Des., 2014, vol. 84, p. 473. doi 10.1111/cbdd.12336.

12. Li, Y.Y., Fang, J., and Ao, G.Z., Expert Opin. Ther. Pat., 2016, vol. 1, p. 14. doi 10.1080/ 13543776.2017.1272572

13. Lin, L., Betsuyaku, T., Heimbach, L., Li, N., Rubenstein, D., Shapiro, S.D., An, L., Giudice, G.J., Diaz, L.A., Senior, R.M., and Liu, Z., Matrix Biol., 2012, vol. 31, p. 38. doi 10.1016/j.matbio.2011.09.003 
14. Moroy, G., Alix, A.J., Sapi, J., Hornebeck, W., and Bourguet, E., Anticancer Agents Med. Chem., 2012, vol. 12, p. 565. doi 10.2174/187152012800617696

15. Allen, F.H., Kennard, O., Watson, D.G., Brammer, L., Orpen, A.G., and Taylor, R., J. Chem. Soc. Perkin Trans. 2, 1987, vol. 2, no. 12, p. 1. doi 10.1039/P298700000S1

16. Sielaff, F., Than, M.E., Bevec, D., Lindberg, I., and Steinmetzer, T., Bioorg. Med. Chem. Lett., 2011, vol. 21, p. 836. doi 10.1016/j.bmcl.2010.11.092

17. Navia, M.A., McKeever, B.M., Springer, J.P., Lin, T.Y., Williams, H.R., Fluder, E.M., Dorn, C.P., and Hoog- steen, K., Proc. Nat. Acad. Sci. USA, 1989. 86, p. 7. doi 10.1073/pnas.86.1.7

18. Sheldrick, G.M., Acta Crystaollogr. (A), 2008, vol. 64, p. 112. doi $10.1107 / \mathrm{s} 0108767307043930$

19. Sheldrick, G.M., SHELXL-97. Program for the Refinement of Crystal Structures. Göttingen University, Göttingen, Germany, 1997.

20. Schepetkin, I.A., Khlebnikov, A.I., and Quinn, M.T., J. Med. Chem., 2007, vol. 50, p. 4928. doi 10.1021/ jm070600+ 